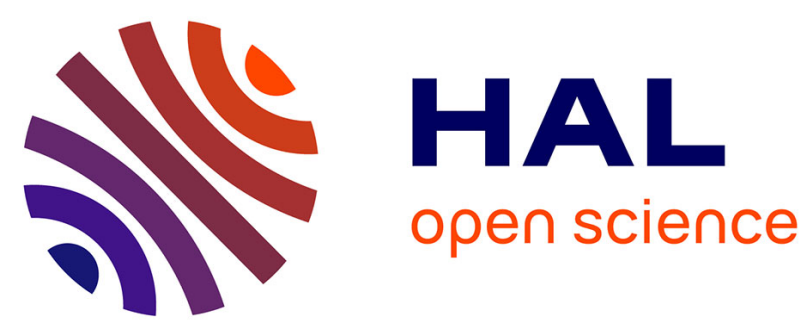

\title{
Software Innovation Dynamics in CMSs and Its Impact on Enterprise Information Systems Development
}

\author{
Andrzej Skulimowski, Inez Badecka
}

\section{To cite this version:}

Andrzej Skulimowski, Inez Badecka. Software Innovation Dynamics in CMSs and Its Impact on Enterprise Information Systems Development. 10th International Conference on Research and Practical Issues of Enterprise Information Systems (CONFENIS), Dec 2016, Vienna, Austria. pp.309-324, 10.1007/978-3-319-49944-4_23. hal-01630527

\section{HAL Id: hal-01630527 \\ https://hal.inria.fr/hal-01630527}

Submitted on 7 Nov 2017

HAL is a multi-disciplinary open access archive for the deposit and dissemination of scientific research documents, whether they are published or not. The documents may come from teaching and research institutions in France or abroad, or from public or private research centers.
L'archive ouverte pluridisciplinaire HAL, est destinée au dépôt et à la diffusion de documents scientifiques de niveau recherche, publiés ou non, émanant des établissements d'enseignement et de recherche français ou étrangers, des laboratoires publics ou privés. 


\title{
Software Innovation Dynamics in CMSs and its Impact on Enterprise Information Systems Development
}

\author{
Andrzej M.J. Skulimowski ${ }^{1,2}$, Inez Badecka ${ }^{2}$ \\ ${ }^{1}$ AGH University of Science and Technology, Chair of Automatic Control and Biomedical \\ Engineering, Decision Science Laboratory, Al. Mickiewicza 30, 30-050 Krakow, Poland \\ ${ }^{2}$ International Centre for Decision Sciences and Forecasting, Progress \& Business \\ Foundation, Lea 12B, 30-048 Kraków, Poland \\ e-mail: ams (at) agh . edu.pl
}

\begin{abstract}
This paper reports the results of a prospective study on information system development trends. It is based on the observation that SMEs seek opportunities to endow their CMS-based IS with CRM, e-commerce and ERP/ERM functionalities. Using publicly-available release data, we investigated the dynamics of subsequent versions and new functionalities of the most popular open-source CMSs - Drupal, Joomla!, and WordPress. Special attention was paid to software innovations that make possible the use of CMS-based applications for typical EIS purposes. The software technology race was modelled by a system of quasi-linear stochastic equations with state variables describing the upgrade generation time. Two such models have been built and compared for the above CMSs. Trend extrapolation with vector autoregression allowed us to predict ERP-related functionality development prospects until 2025. We maintain that the deployment of CMS-based ERP/ERM may have a relevant impact on business models and strategic ICT alignment in SMEs.
\end{abstract}

Keywords. Software evolution, ICT foresight, ERP-CMS, vector autoregression, EIS scenarios, open-source software.

\section{Introduction}

The deployment order and intensity for different information and communication technologies (ICTs) in enterprises is an important issue from the software market point of view. It is also a research question relevant to understanding the technological evolution of Enterprise Information Systems (EISs) and their market development trends. ICT deployment trajectories in large enterprises have been extensively studied by many researchers cf. e.g. [3],[8],[9],[15], who have often referred to them as strategic ICT alignment [4]. However, the ICT investment behaviour of the small and medium sized enterprises (SMEs) is known in far less detail due to the great diversity of SMEs structures and business models and a greater flexibility of SME managerial decisions that often depend on individual preferences. Nevertheless, SMEs are expected to be the fastest growing market for corporate ICT solutions over the next decade [18]. Software market research indicates that the most important ICT functionalities for SMEs are those offered by Web 2.0 CMS technologies. The research presented in 
[20] provided clues that enterprise resource planning (ERP) systems based on the collaboration of developer groups, supported by web browsers and typical CMSs, could become a significant future trend among SMEs. This conjecture has been confirmed by a needs analysis performed among enterprises during the initial phase of a foresight project [18]. In contrast to big companies, which use large and complex multi-module ERP applications such as SAP, SMEs widely use in-company online solutions [6] based on open-source CMSs, for cost reasons. Further studies conducted within the above project [18] showed that small and micro enterprises in particular intend to further invest in the development of such systems. This trend can also be regarded as a manifestation of the business model alignment to software evolution in the context of syntactic integration of different enterprise software systems [16].

As a sample data set, this paper uses the CMS versions and the mutual relations of ERP and CMS development trends (ERP-CMS) gathered in the project SCETIST [18]. Partial results concerning ERP-CMS have been presented in [19] and [20]. A general technology race model for the most common open-source CMSs Drupal, Joomla! and WordPress was proposed in the form of a quasi-linear system of stochastic equations describing software evolution trajectories. The state variables are time lapses between generating two subsequent software releases of each CMS under consideration. We will construct, analyse and compare two specific models of upgrade generation processes for the three CMSs. These models correspond to software development scenarios that differ on factors taken into account when making a decision to produce a new release that brings an essential software improvement.

Both models take into account the emergence of essential new functionalities in the new releases. The first model refers to a situation where the innovation leader is fully independent in shaping its development strategy, without taking into account the progress made by its main competitors. The form of this model is justified by historical data concerning the CMS innovation leader Drupal which point out that the novelties offered in new releases of WordPress and Joomla! have not influenced the main line of Drupal development so far. The second model assumes a symmetric impact of innovation among all software providers.

The model coefficients are calibrated based on historical data published by the CMS providers on their websites. However, the coefficients of the second model that describe the future dependence of the current innovation leader on the other systems after it loses leadership could not be estimated as such a situation has never occurred before. Instead, these coefficients are assumed to be similar to the reactions of other systems on the competitor's advantages. They are then used in calculation of future release generation forecasts together with estimated coefficients describing other CMSs. Thus, the main goal of this paper is to apply the above foresight model composed of two long-term software development scenarios to provide clues to SME managers as regards the viability of the open source software they use as a seed information technology [19] in their enterprises.

Trend extrapolation with vector autoregression has made it possible to construct two scenarios of ERP-related functionality development prospects with open source CMSs until 2025 and beyond, related to the above models. The technological trajectories thus generated show surprising future behaviour, indicating changes in innovation leadership. According to these forecasts, Drupal, the current innovation leader, may lose its market position if it does not take into account the innovation potential of its 
competitors. In the final section, we will discuss the related basic business scenarios of SMEs as well as the synergy with other EIS development trends. We conclude that the open-source CMSs are viable enough to capture a considerable portion of the ERP for SMEs market over the next decade. Consequently, vendors and authorized resellers of popular ERP systems may lose some of their revenue to CMS developers.

ERP based on CMS and web portal technologies (in this paper referred to as CMS ERP, cf. also [12],[25]) is an application that contains an administrative panel endowed with a complex hierarchy of user authorisation levels. It can be used to exchange information within the enterprise and externally, and manage its resources. The ERPCMS emerged as a result of including software development tools in advanced CMSs, making possible the implementation of resource-management processes.

To conclude this section, in Box 1 below we recall a few basic notions related to EISs used in further parts of this paper.

Box 1. Basic notions relevant to Enterprise Information System development trends.

EIS: Enterprise Information System is a key notion used in this paper. EIS is a broad class of business software facilitating information integration within an enterprise and its exchange with external agents (clents, suppliers, business partners, authorities etc.).

EAI: Enterprise Application Integration (EAI) is the process of sharing and linking different information and business processes in an organization [4],[14]. As a result of EAI, most ICTsupported business processes are entirely controlled by a unified application. The same application ensures access to all or most enterprise databases and an appropriate common data management system. The interconnection of all the organisational data forms a basis for implementing efficient decision support systems [1].

ERP: Enterprise Resource Planning is an ICT-based integration of business processes in an organisation. Usually, ERP is implemented as an information system with a modular architecture, where each module manages a specific area of activity. The same shortcut, ERP, or 'ERP system', also refers to an integrated software solution that implements the above process [3].

ERM: Enterprise Resource Management is a term which refers to ERP in a more general setting and gradually replaces the ERP term. It reflects the extension of enterprise application integration beyond resource planning. The notion of resources is more general and covers any information managed by the company and its intangible assets. One of the ultimate goals of ERM is to provide decision support to enterprise management [3], [10].

CMS: A Content Management System is a web application (or a set of applications) that facilitates the development of a website, and other forms of web-based information systems by non-technical content editors. CMSs are increasingly including modules and functionalities that support enterprise management [16].

\section{Basic Properties and a Review of Popular Open-Source CMSs}

The main idea of a typical CMS is to allow a non-expert user to edit the content of a website and modify its design via an easy-to-use user interface, called an administration panel. The main task of CMS platforms is to separate the information content of the website from the technical aspects of its appearance. CMSs generate web pages automatically or semi-automatically. The information entered by an 
authorised editor is stored in a database. The CMS generates a dynamic website based on the content of this database and on a selected template. This allows for more flexible and convenient content management than with static HTML files. Thanks to this approach, the publishing of web pages has become much simpler than in the past.

\subsection{A review of most popular CMS}

Popularity scores of CMSs are provided by different web application market watchers [2], where the number of installations varying in time can be found, with the demarcation of specific industries or sectors of use. The three systems selected for detailed analysis in this paper cover most of the CMS market. They are briefly outlined below.

WordPress [23] has become the most popular content management system during the current decade. It is written in PHP and uses a MySQL database. WordPress is distributed under the GNU - General Public License. As the official successor of the b2/cafélog blog system, WordPress is the most popular and user-friendly system for blogging. Among the many CMSs, WordPress appears as the easiest to install, operate and configure. Due to its growing range of functionalities and ease of use, WordPress is increasingly popular in the business sector, as well as with entertainment and social networking sites. With its recent enterprise-oriented premium spin-out, WordPress Vip, the platform fulfils the early predictions concerning the CMS development trends contained in [20], cf. also https://vip.wordpress.com/2014/05/22/ enterprisewordpress-content-trends.

The Joomla! project was founded in August 2005 by the team who developed Mambo, a predecessor of Joomla [11]. After the development of Mambo was abandoned, future versions have appeared, such as Aliro (http://www.aliro.org), Lanius CMS (http://www.laniuscms.org), Elxis (http://www.elxis.org) etc. Joomla has a modular structure, which means that each new feature of the system is added as an additional module. This allows enterprise users to easily extend the usability of the system into the e-commerce, frequently starting from product catalogues, as well as to use its CRM and ERP/ERM functionalities.

Drupal [7] is a CMS that allows its users to easily publish, manage and organise web content. It is equipped with functionalities that include environments for collaborative work on projects, file exchange and much more. An important feature of Drupal is its system of modules and taxonomy. The latter allows the users to organise web contents according to predefined categories. Drupal plays an important role in this paper due to its two exceptional features:

- In recent years, Drupal has led the CMS field in terms of implementing innovations in its releases,

- Drupal is the leader in offering ERP-related modules [7].

Overall, Drupal is equipped with many special tools that are useful in the business sector. The platform is scalable and can be used to build enterprise information systems of any size. Moreover, Drupal strives to simplify its use via 'Drupal Open Enterprise' (https://www.drupal.org/project/openenterprise). 


\subsection{The technological evolution of CMSs}

The Fig. 1 summarises, in the form of a timeline, the history of versions, milestones, and functionalities of the most popular CMSs covered in the preceding section: Wordpress, Joomla and Drupal. The data has been gathered in [18] and [20] and verified based on the recent information provided by the software suppliers.

The historical data characterising the relative innovativeness of the selected CMSs represented in Fig. 1 and used to build the models presented in the next section is based on [12]. The identification of relevant functionalities and milestones illustrated in the above timeline, together with the version data, forms the basis on which to build both technological evolution models.

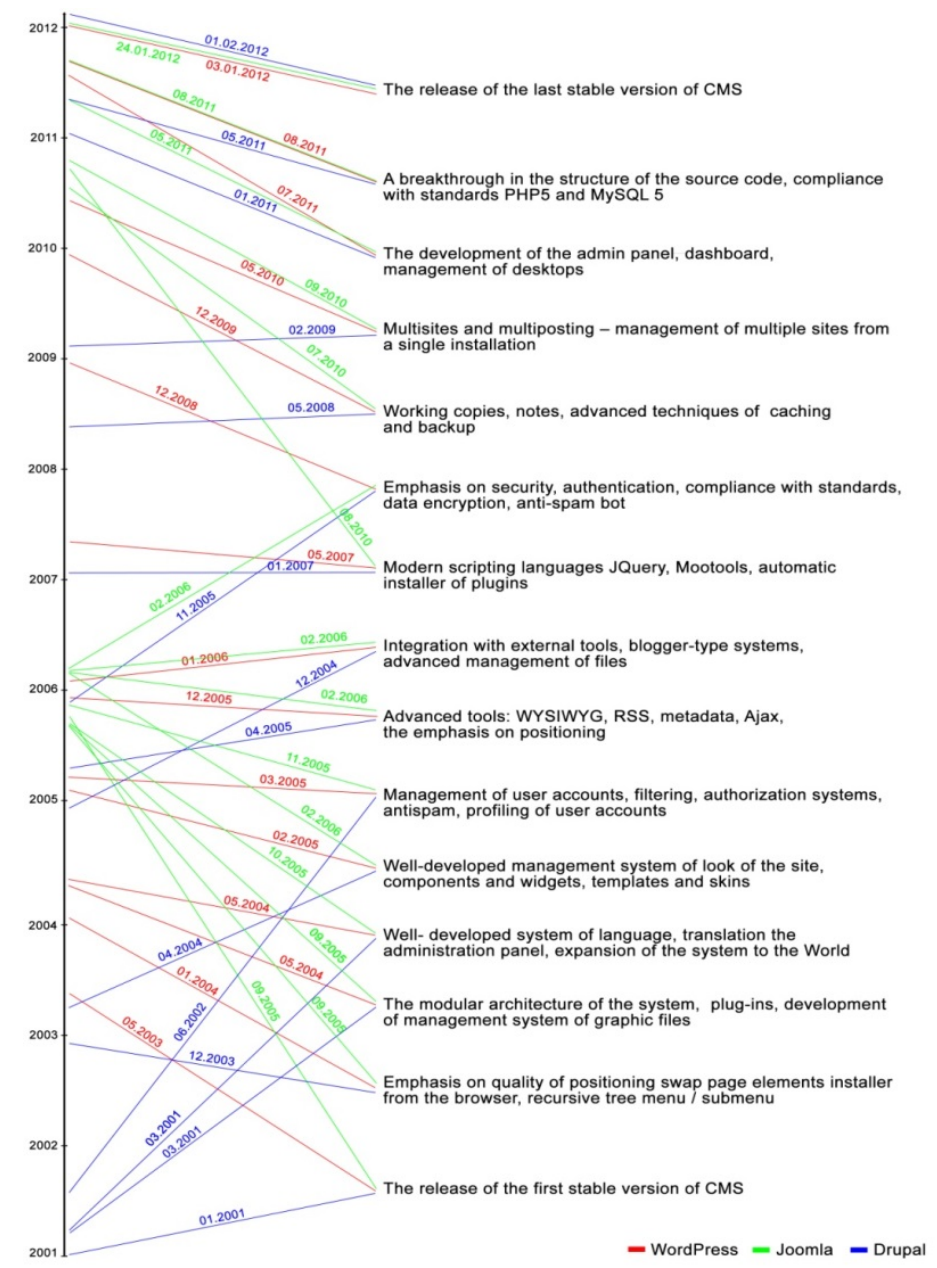

Fig. 1. A timeline of the development of the three most frequently-used open source CMSs. The coloured lines point out the release of main functionalities of these CMSs on the time scale. 


\section{Two Scenarios of Technological Evolution}

There are various models which describe the generation of software innovation, however, none of them is widely-accepted. General discrete-time innovation diffusion models usually refer to the well-known Norton-Bass model [10], [12]. When applied to open-source software, in the absence of price equilibria, the major role played by essential technological progress must be taken into account. This is partly included in the famous eight Lehman software evolution laws, cf. e.g. [24], pp.49-53, 58-61, although they do not sufficiently refer to the emerging oligopolistic structure of open source software supply. Therefore, in [19],[20] we proposed a new class of stochastic models, suitable for information systems evolution modelling, including the open source CMS. The dependent variable is the time of market release of a relevant innovation by the developers. Explanatory variables are time lags between consecutive releases of the same as well as of other systems. In [19] we compared models based on major version releases and taking into account selected essential functionalities only.

Following the research reported in [19], this paper extends the scope of modelling to include models based on analogies, to be applied in case where no or too few observations are available to use statistical fitting techniques. This allows us to construct a realistic forecasting model for a situation where the hitherto market leader may lose its leadership during the forecast period.

In both models presented in the following subsections 3.1 and 3.2 we take into account only the releases of each software system that bring considerable innovation of relevance to the user community. Specifically, for a given software system $S$ we assume that the time lags from recent releases of other systems are less important to explain the evolution of this kind of software than the dates of the introduction of relevant functionalities, which system $S$ does not yet possess. Based on empirical evidence, in the first model we have additionally assumed that the technological leader (Drupal) develops its system autonomously, i.e. without taking into account the innovations introduced by systems that have been less advanced so far. Another development scenario, which does not admit this assumption and treats all systems as dependent on the innovations introduced by other developer teams, yields the second model. These models will generate two CMS and ERP-CMS development and deployment scenarios, termed Scenario A and Scenario B, respectively. Both models and the corresponding scenarios are investigated are compared in Section 4.

As already mentioned, the coeffcients and their goodness of fit of the second model (Scenario B) could not be determined properly because the situation where the mutual dependence of the leading system on the others did not occur in the past. However, simulations of such behavior based on analogy to other developer teams are possible and are crucial in building the second model. The goodness of fit of a simulated Model 2 may be worse than in the case of Model 1 since the estimation of missing interdependence parameters is based on analogies, and not on the extrapolation. However, the study of the second model has an exploratory character while its lower accuracy will be compensated by a better coverage of possible states of the future.

In the following sections we will present the assumptions that led to the formulation of both models, the forecasts that they generate, the impact on the development of CMS technology and the resulting conclusions. 


\subsection{Innovation forecasts in Scenario A}

Let $x(i), y(i)$, and $z(i)$ denote time intervals between next (i-th) essential system improvement, for WordPress, Joomla! and Drupal, respectively. We can then examine the relationship between the time of the next improvement introduction by a particular development team and the frequency of similar innovations in the past, created by the developer teams of all above CMSs.

We assume that the time interval of the subsequent system improvement depends linearly on the $n-1$ previous time intervals of system improvements as well as on the frequency of the improvements emerging in other systems. Motivated by a timeline chart analysis (Fig. 1), which showed Drupal leading in the implementation of all previous innovations, the first scenario will additionally assume that the Drupal development team operates autonomously, without taking into account subsequent versions of WordPress and Joomla. The innovations introduced by the latter two are already present in Drupal. These innovations are not influenced by a competitive pressure on the Drupal development team (such pressure may, however, result from introducing marketing or organisational innovations, which are not necessarily linked with the technological innovation introduced by the successive versions of software). These assumptions lead to the formulation of the following model of innovation creation in the three analysed systems (1), which is an extension of that proposed in [19]:

$$
\begin{gathered}
x(k+1)=a_{1,1} x(k)+a_{1,2} x(k-1)+\ldots+a_{1 n} x(k-n+1)+b_{1,2} v_{1,2}(k)+b_{1,3} v_{1,3}(k)+c_{1} \\
y(k+1)=a_{2,1} y(k)+a_{2,2} y(k-1)+\ldots+a_{2 n} y(k-n+1)+b_{2,1} v_{2,1}(k)+b_{2,3} v_{2,3}(k)+c_{2} \\
z(k+1)=a_{31} z(k)+a_{32} z(k-1)+\ldots+a_{3 n} z(k-n+1)+c_{3}
\end{gathered}
$$

where:

$v_{12}(k)$ - average frequency of introducing a new functionality of Joomla calculated on the basis of $P_{1,2}(\mathrm{k})$ time intervals between essential releases of this system directly preceding the $k$-th essential functionality of WordPress;

$v_{21}(k)$ - average frequency of introducing of a new functionality of WordPress calculated on the basis of $P_{2,1}(k)$ time intervals between essential releases of this system directly preceding the $k$-th essential functionality of Joomla

$v_{j, 3}(k)$, for $j=1,2$ - average frequency of introducing of a new version of Drupal calculated on the basis of $P_{j, 3}(k)$ time intervals between essential releases of this system directly preceding the $k$-th essential functionality of WordPress - for $j=1$, and Joomla - for $j=2$.

Based on numerical experiments, in the above model we will admit a further simplifying assumption, namely as $P_{i, j}(k)$, we will take the maximum value $P_{i, j}(k)=3$, for $i=1,2, j=1,2,3, i \neq j$ which, however, will allow us to obtain a sufficient statistical significance of the model. Furthermore, we will take into account only positive time lags between launching essential functionalities, i.e. we assume that exclusively the technological arrears play a motivating role for the developer teams.

Let $a_{j 1}, a_{j 2}, \ldots, a_{j n}$ denote the direction coefficients of the regression equation for the dependence of the time interval of the $j$-th system new functionality appearance on the previous frequencies of innovation introduced in the same system, i.e. WordPress, Joomla and Drupal, respectively, for $j=1,2,3$. The $b_{i, j}$ for $i=1,2, j=1,2,3$, and $i \neq j$, 
denote the coefficients of the linear regression functions that describe the following dependencies:

- for $i=1, j=2,3, b_{i, j}$ is the coefficient of multivariate linear regression which explains $x(k)$ with $v_{1, j}(k)$,

- for $i=2, j=1, b_{i, j}$ is the coefficient of multivariate linear regression which explains $y(k)$ with $v_{2,1}(k)$ and for $i=2, j=3, b_{i, j}$ explains $y(k)$ with $v_{1,3}(k)$.

After finding the coefficients of (1) with the least squares method (LSM), we will get the regression function relating the expected time of a new innovation release by each system - as dependent variables - to the average time lags between the introduction of technological innovations in all systems. The lags are calculated to the releases directly preceding the latest innovation in the $i$-th system, prior to the $k$-th improvement of the system described by the equations (1a) or (1b). The trend drift coefficients $c_{i}, i=1,2,3$ should vanish after the Model (1) for autoregresssion purposes was integrated sufficiently many times to yield a stationary time series for each system. However, they re-appear when calculating the forecasts for the original time series.

The equations (1a), (1b) take into account the pressure of competition. According to the initial assumption, equation (1c) describes time lags between subsequent Drupal improvements only.

It turned out impossible to find a statistically significant model where the variables were simple time lags between innovations. However, a stationary time series and, at the same time, significant regression functions could be found when averaging the variables three times. From the observation that, by the definition of variables as time lags, they possess the property (2),

$$
x(t)-x(t-n)=[(x(t)-x(t-1)]+[x(t-1)-x(t-2)]+\ldots+[x(t-n+1)-x(t-n)],
$$

it follows that the above averaging operation is equivalent to the integration of the original time series.

The statistical significance of both models was investigated with the $F$ (FisherSnedecor, cf. e.g. [21]) and goodness of fit tests with the determination coefficient $R^{2}$. Tests confirmed the significance of the calculation results for triple averaged data, which corresponds to the case where all variables characterising the frequency of innovations are calculated as an average of three time lags between essential new functionalities in all systems modeled.

Finally, the forecasting model in Scenario A is provided in eq. (3) below.

$$
\begin{gathered}
x(k+1)=0.1521 x(k)+0.6284 v_{1,2}(k)+2.9762 v_{1,3}(k)+8.2919 \\
y(k+1)=-0.3679 y(k)+2.9762 v_{2,1}(k)-2.0860 v_{2,3}(k)-18.3726 \\
z(k+1)=1.1662 z(k)-0.6892 z(k-1)+4.6565
\end{gathered}
$$

As the significance of the regression function does not guarantee that the coefficients are significant, we calculated the confidence intervals for each of them. Then, to complete the usual statistical analysis of forecasted values, we calculated the confidence intervals for the number of innovations. The forecasting procedure was pursued until the last release of the most slowly evolving system reached the year 2025, i.e. the foresight horizon of the project SCETIST [18].

The forecasting results for the horizons 2025 and 2030 are provided in Table 1, while the forecasts of the number of implemented innovations are visualised in Fig. 2. 
Table 1 . The forecasts of implemented innovations in system releases and their confidence intervals $(p=0.95)$ for the Scenario A

\begin{tabular}{ccc|cccc|ccc}
\hline \multicolumn{3}{c}{ Drupal } & \multicolumn{4}{c}{ WordPress } & \multicolumn{3}{c}{ Joomla! } \\
\hline $\begin{array}{c}\text { Forecast } \\
\text { horizon }\end{array}$ & $\begin{array}{c}\text { Number of Confidence } \\
\text { innovations }\end{array}$ & $\begin{array}{c}\text { Forecast } \\
\text { interval } \\
\text { horizon }\end{array}$ & \multicolumn{2}{c}{$\begin{array}{c}\text { Number of } \\
\text { innovations }\end{array}$} & $\begin{array}{c}\text { Confidence } \\
\text { interval }\end{array}$ & $\begin{array}{c}\text { Forecast } \\
\text { horizon }\end{array}$ & $\begin{array}{c}\text { Number of Confidence } \\
\text { innovations }\end{array}$ & interval \\
\hline $2025-01$ & 34 & $(27,42)$ & $2025-06$ & 33 & $(28,39)$ & $2025-12$ & 34 & $(27,42)$ \\
$2030-05$ & 42 & $(36,52)$ & $2030-01$ & 45 & $(38,52)$ & $2030-07$ & 39 & $(32,49)$ \\
\hline
\end{tabular}

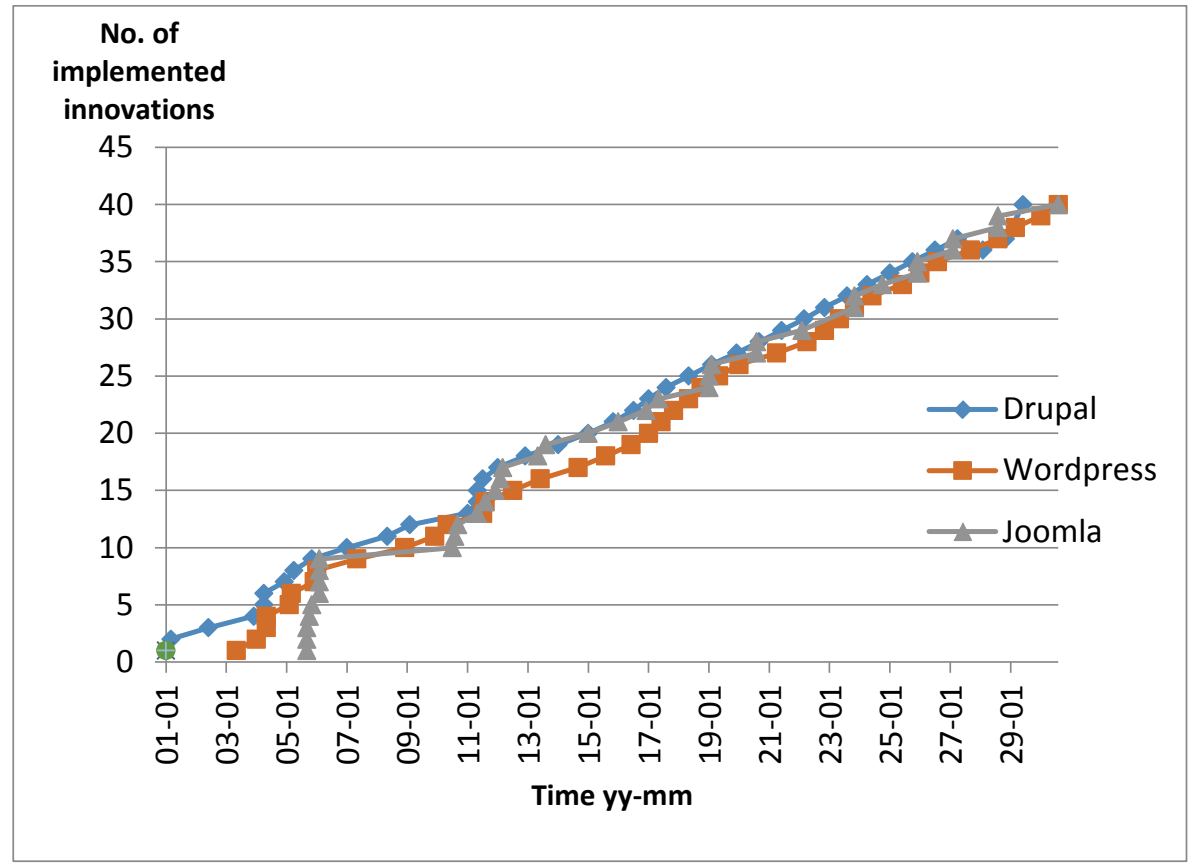

Fig. 2. Forecasted number of implemented innovations by each of the systems in Scenario A.

The chart shows that Drupal, which was assumed to develop autonomously, will temporarily lose its leadership twice. Joomla will catch up with Drupal within a few years and the development of both systems will follow the same trajectory until 2025. Both systems will overtake Wordpress. In about 10 years from now all systems will converge, i.e. the number of implemented innovations in WordPress, Joomla and Drupal will be practically equal. This may ultimately result in mergers or otherwise elimination of some systems from the market as the community of developers will not differentiate them anymore. The simulation points out that this situation may change until 2030. However, the confidence intervals of the forecasted quantities will already be rather large in 2025 and grow further beyond this time horizon (cf. Table 1) while the longer-term forecasts may be disturbed by new factors and phenomena that did not appear by now and were not included in model (1). 


\subsection{Innovation trajectories in Scenario B}

Let us recall that the Scenario B differs from the Scenario A on the assumption that all systems, including the current innovation leader, take into account the new functionalities introduced in the software releases of their competitors. Their decisions concerning the time of new version releases are modified quasi-linearly according to the time elapsed since the new functionalities were launched by other systems. To build a CMS forecasting model with this assumption, we will use the same essential functional improvements that have been identified for the Model 1 and Scenario A, cf. eq. (1), Fig. 2. The interdependence coefficients between competing systems are calculated based on the time lags (in months) between subsequent ( $i$-th) essential system improvements. The assumptions concerning the variables are the same as in Model 1, in particular only positive lags between the introduction of new functionalities are taken into account. This led us to the formulation of an extended model of essential innovation generation in WordPress, Joomla, and Drupal, described by eq. (4).

$$
\begin{aligned}
& x(k+1)=a_{1,1} x(k)+a_{1,2} x(k-1)+\ldots+a_{1 n} x(k-n+1)+b_{1,2} v_{1,2}(k)+b_{1,3} v_{1,3}(k)+c_{1} \\
& y(k+1)=a_{2,1} y(k)+a_{2,2} y(k-1)+\ldots+a_{2 n} y(k-n+1)+b_{2,1} v_{2,1}(k)+b_{2,3} v_{2,3}(k)+c_{2} \\
& z(k+1)=a_{31} z(k)+a_{32} z(k-1)+\ldots+a_{3 n} z(k-n+1)+b_{3,1} v_{3,1}(k)+b_{3,2} v_{3,2}(k)+c_{3}
\end{aligned}
$$

The notation is similar to that used in Scenario A and Model (1). The new coefficients of the Model 2, $v_{3, j}(k)$, for $j=1,2$, denote the average frequency of introducing a new version of Wordpress $(j=1)$ or Joomla $(j=2)$ calculated on the basis of $P_{3, j}(k)$ time intervals between essential releases of the corresponding system directly preceding the $k$-th essential functionality of Drupal. The other difference consists in the fact that the coefficients $b_{3,1}$ and $b_{3,2}$ could not be estimated with multivariate regression because during the observation period (2004-2012) Drupal was always a leader in launching new functionalities, so the variables $v_{3,1}(k)$ and $v_{3,2}(k)$ were identically equal to 0 . To simulate the reaction of the Drupal's team on a potential different situation that may occur in the future, $b_{3,1}$ and $b_{3,2}$ were assumed equal to 1.5 and 0.7 , respectively. These values resulted from the averaging of the reactions of WordPress and Joomla teams on the past Drupal's advantages, taking into account the mean values of the variables $x, y$, and $z$. The subjectivity of this assumption illustrates the difference between the forecasting and foresight methodology: foresight approaches make it possible to explore future in the situation where no extrapolation of the past is possible, based on heuristic observations and assumptions. Analogy-based reasoning is the only way to investigate the phenomena that have never occurred before.

The triple averaging of input data yielded again the best outcomes. The coefficients of the second model are provided in eq. (5) below.

$$
\begin{gathered}
x(k+1)=0.1521 x(k)+0.6284 v_{1,2}(k)+2.9762 v_{1,3}(k)+8.2919 \\
y(k+1)=-0.3679 y(k)+2.9762 v_{2,1}(k)-2.0860 v_{2,3}(k)-18.3726 \\
z(k+1)=1.1662 z(k)-0.6892 z(k-1)+1.5 v_{3,1}(k)+0.7 v_{3,2}(k)+4.6565
\end{gathered}
$$

The significance of Model (5) is the same as of Model (1) during the observation period and it is undefined during the forecasting period. The occurrence of any event 
that causes non-zero values of $v_{3,1}(k)$ and $v_{3,2}(k)$ would make it possible to use supervisory learning techniques to update the coefficients $b_{3,1}$ and $b_{3,2}$. The results of the CMS innovation implementation forecasts in Scenario B are shown in Fig.3.

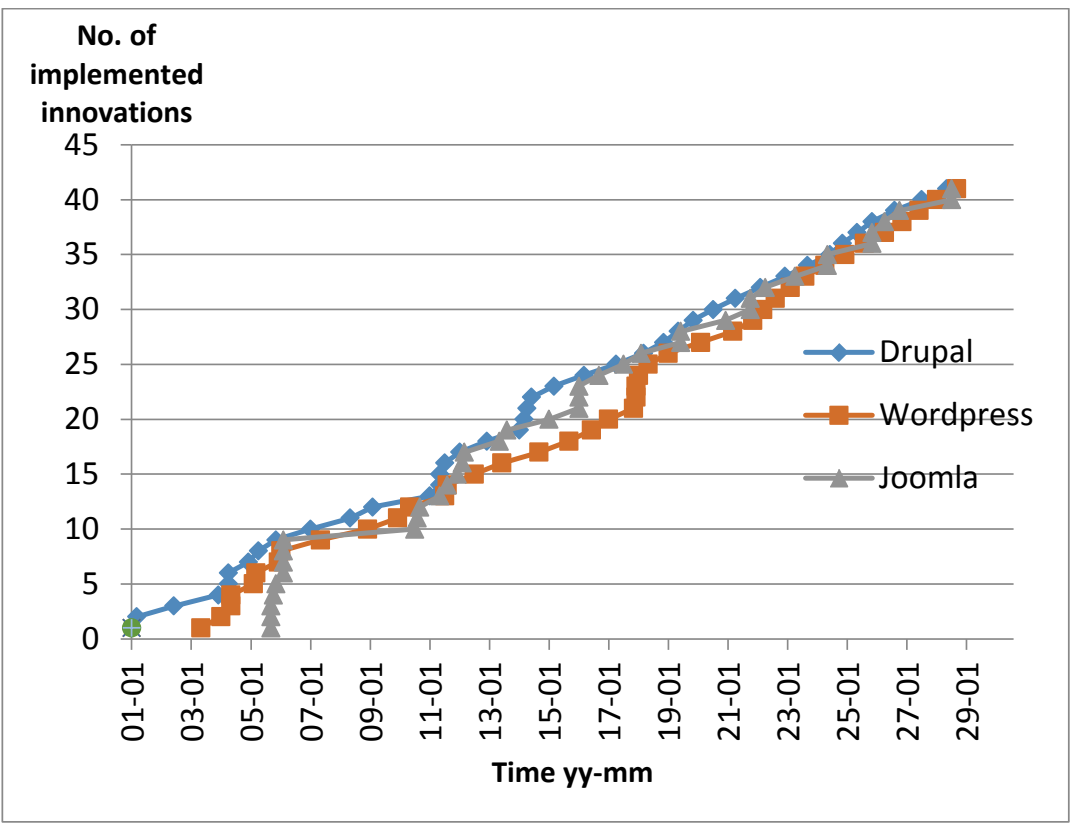

Fig. 3. Forecasted number of innovations for the three CMS in Model 2 - Scenario B

The ability to observe the other systems and react gives the Drupal team a development impulse every time it is surpassed by Joomla. In this scenario WordPress stays initially behind, but at the end of the forecasting period the functionalities of all systems differ only slightly. Similarly as in Scenario A, it gives again clues as regards potential mergers or transformations of main open source CMSs during the next decade. The evolution of Internet, the emergence of new business models, and new information technologies may additionally contribute to an end of CMS era.

The confidence intervals for the quantities of implemented innovations by the two forecasting horizons 2025 and 2030 are presented in Table 2 below. The assumed coefficients $b_{3,1}$ and $b_{3,2}$ are regarded deterministic and do not influence the stochastic properties of Model (5).

Table 2. The forecasts of implemented innovations in system releases and their confidence intervals $(p=0.95)$ for the Scenario B

\begin{tabular}{ccc|ccc|ccc}
\hline \multicolumn{3}{c}{ Drupal } & \multicolumn{3}{c}{ WordPress } & \multicolumn{3}{c}{ Joomla! } \\
\hline $\begin{array}{c}\text { Forecast } \\
\text { horizon }\end{array}$ & $\begin{array}{c}\text { Number of Confidence } \\
\text { innovations }\end{array}$ & $\begin{array}{c}\text { Forecast } \\
\text { interval }\end{array}$ & $\begin{array}{c}\text { Number of Confidence } \\
\text { horizon }\end{array}$ & $\begin{array}{c}\text { Forecast } \\
\text { innovations } \\
\text { honterval }\end{array}$ & $\begin{array}{c}\text { Number of Confidence } \\
\text { horizon }\end{array}$ & innovations & interval \\
\hline $2025-05$ & 35 & $(29,41)$ & $2025-08$ & 36 & $(32,41)$ & $2025-11$ & 36 & $(29,42)$ \\
$2030-07$ & 44 & $(36,53)$ & $2030-03$ & 43 & $(37,50)$ & $2031-11$ & 44 & $(36,54)$ \\
\hline
\end{tabular}




\section{A Comparison of Scenarios A and B}

A comparison of both CMS evolution scenarios is shown in Table 3 below. It turns out that there is no considerable difference in CMS innovative behavior between the scenarios, with some more activity in Scenario B. As the only exception, WordPress performs worse on about $4 \%$ in Scenario B in 2030. The ability to consider technological and market signals from the other two systems allowed Drupal to generate slightly more (about 5\% in 2030) new essential functionalities in its releases. Without this ability Drupal will loose its leadership to WordPress until 2030. More active Drupal in Scenario B boosts the innovativeness of Joomla - its number of essential innovation rises on 5 (or on 12\%) until 2030. A better performance of Joomla is accompanied by a loss of the innovateveness of WordPress between 2025 and 2030.

The convergence trend is more salient in Scenario $B$, where the number of functionalities of all systems in 2025 and 2030 is almost the same.

Table 3. Expected number of essential innovations in the CMS releases until 2030 - a scenario comparison

\begin{tabular}{c|cc|cc|cc}
\hline \multicolumn{4}{c}{ Drupal } & \multicolumn{2}{c}{ WordPress } & \multicolumn{2}{c}{ Joomla! } \\
\hline Forecast horizon & Scenario A & Scenario B & Scenario A & Scenario B & Scenario A & Scenario B \\
\hline 2020 & 28 & 30 & 26 & 27 & 27 & 29 \\
2025 & 34 & 35 & 33 & 36 & 34 & 36 \\
2030 & 42 & 44 & 45 & 43 & 39 & 44 \\
\hline
\end{tabular}

Another comparison is provided in Table 4 which shows the value of the indicator "CMS innovativeness growth index" defined in [19]. This indicator ( $5^{\text {th }}$ row in Table 4) is calculated as the ratio of the forecasted yearly average number of innovations during the period of $2012-2025$ ( $4^{\text {th }}$ row in Table 4 ) and the actual average value observed during the period of 2005-2012 ( $3^{\text {rd }}$ row).

Table 4. Number of innovations in CMS releases until 2025 - Scenarios A and B compared

\begin{tabular}{lccccccc}
\hline & \multicolumn{2}{c}{ Drupal } & \multicolumn{2}{c}{ WordPress } & \multicolumn{2}{c}{ Joomla! } \\
\cline { 2 - 7 } & \multicolumn{2}{c}{ Scenario A } & Scenario B & Scenario A & Scenario B & Scenario A Scenario B \\
\hline No. of innovations in 2005-2012 & 10 & 9 & 10 & 10 & 14 & 14 \\
No. of innovations in 2012-2025 & 17 & 20 & 17 & 21 & 18 & 22 \\
Mean innovations no. in 2005-2012 & 1.43 & 1.29 & 1.43 & 1.43 & 2 & 2 \\
Mean innovations no. in 2012-2025 & 1.31 & 1.53 & 1.53 & 1.62 & 1.38 & 1.69 \\
Average innovation growth & 0.92 & 1.20 & 1.07 & 1.13 & 0.69 & 0,85 \\
\hline
\end{tabular}

The above results show that the assumption concerning the market leader behavior that differentiate both scenarios may have a remarkable influence on the innovativeness of all the developer teams concerned. It turns out that a 'weaker' leader that follows its own development strategy and does not react to its competitors' achievements suppresses the innovative activity of all market players. On the contrary, its activity may increase the market strength of all open source CMS suppliers and reduces the differences between their products and innovativeness of developer teams. 


\subsection{Future features and functionalities of CMSs}

An important issue that has not been explained yet is the transition between quantitative innovation characteristics and a concrete sequence of innovations that will be created by each of the CMS development teams. A partial answer can be derived from development plans that, however, are announced for a relatively short period of for one or two years only [7]. Other clues are given by the version and functionality analysis performed in Sec. 2.2 and by both innovation development models presented in Secs. 3.1, 3.2 and 4. It follows that Drupal plays the role of leading innovator, while the other systems follow its functionalities with some delay. Taking into account that Drupal has already entered the ERP/ERM market and plans further expansion in enterprise software sector, it can be concluded that the other systems will follow suit during the next decade.

In addition, all the above-analysed CMS developers pay much attention to research, which allows them to quickly implement new technologies. Below is a (nonexhaustive) list of ideas, functionalities, and new modules that are either in the process of being tested or are planned for implementation during the next few years (cf. the CMS web sites [7],[11],[23]). Some of them will support ERP functionalities or meet general enterprise needs. A few new ERP-related features, namely innovation support modules as well as HR management systems, have been pointed out as relevant by the respondents of the Delphi survey performed within the project SCETIST [18]. They are also included in the list below.

- Cognitive user models allowing the system to learn users interact with the website and the applications installed there. These models will be further developed towards intelligent recommendation systems.

- General enterprise managerial decision support modules using integrated CMS-supported databases containing data provided by other subsystems [1].

- HR management.

- Quality management modules - different versions according to the sector needs and ISO, EMAS, or other quality norms implemented.

- Template builder - easy creation of templates from the administration panel.

- In-company innovation support systems.

The approach applied in this paper yields models that put emphasis on the overall number of functionalities implemented in software systems. They do not yet allow us to determine the sequence of implementation of specific features. However, the latter may be estimated based on development roadmaps published on CMS websites, on research of market expectations and scenarios of software use, and on general software evolution trends. A more detailed study of technological trajectories of ERPCMS and enterprise decision support systems will be a subject of further research.

\section{Conclusions}

In this study, we described the foundations of an important software development trend, namely enterprise application integration (EAI) based on the gradual expansion 
of the scope of applications covered by a company's CMS functionalities. We conducted an overview and comparison of the most popular open source CMSs used primarily, but not exclusively, by SMEs. We maintain that this trend will bring new technological opportunities to SMEs in particular. Using open-source software modules, SMEs will be able to cover more areas of their commercial activity with modern ICT solutions. This trend will interfere with the "going mobile" and "moving to the cloud" trends that are influencing all enterprises, but are of particular importance to small and micro companies. Specifically, web-based ERP/ERM applications are in a better position to include mobile technologies than offline systems. This is the case of applications built with CMS technology, both open-source and commercial.

While CMS-based systems will expand into the cloud and mobile worlds, according to the Delphi survey performed in [18], the accounting software used in SMEs in the perspective of 2025 will still resist full integration in cloud-based systems. This is partly due to the traditional vigilance and concern that entrepreneurs have as regards revealing their financial data in the public space, despite the fact that ICT security has been steadily improving. This contradicts the widely-held belief that a vast majority of enterprises will gradually implement professional ERP software built around accounting, inventory and sales modules. Thus, accounting and personal record files may remain the core of traditional ERP systems. However, this could vary from country to country [15] and may depend on the economic sector.

Finally, let us observe that the methodology presented in Secs. 2, 3 and 4 can be used to investigate the technology race between companies that regularly release new versions of non-CMS software products. A necessary prerequisite is that the market for the product under study is oligopolistic, with a reasonably small number of suppliers (say, less than 20) that enables building and constructively analyzing a model of type (1). Some hints regarding sample-based generalization strategies of software-related models are provided in [22]. While many open source applications and programming environments fulfill this assumption well, the approach presented in this paper is not restricted to software and may be used to forecast the development of electronic components, cars, and other products and technologies.

Acknowledgement. This research was supported by the research project "Scenarios and Development Trends of Selected Information Society Technologies until 2025" (SCETIST) co-financed by the ERDF, Contract No. WND-POIG.01.01.01-00-021/09.

\section{References}

[1]. Asprey, L.; Middleton, M.R.: Integrated document management for decision support. In: Burstein, F.; Holsapple, C. (Eds.), Handbook on DSS., Vol.1, pp. 191--206 (2008)

[2]. Builtwith website (web technology lookup), trends.builtwith.com/cms. Accessed 07/2016

[3]. Castellina, N.: SaaS and Cloud ERP trends, observations, and performances. Aberdeen Group (2011)

[4]. Cataldo, A.; McQueen, R.J.; Hardings, J.: Comparing Strategic IT Alignment Versus Process IT Alignment in SMEs, J. Res. Pract. Inf. Technol. 44(1), 43--57 (2012)

[5]. Chia-Chen, Y.; Chiaming, Y.; Jih-Shih, H.: A Web-based CMS/PDM Integration for Product Design and Manufacturing, IEEE Int. Conf. on e-Business Eng., pp. 549--553 (2008) 
[6]. Devos, J.; Landeghem, H.; Deschoolmeester, D.: Using bricolage to facilitate emergent collectives in SMEs. Proc. $6^{\text {th }}$ European Conf. on Inform. Manag. Eval., pp.82--90 (2012)

[7]. Drupal web site. Drupal Foundation, www.drupal.org, Accessed 07/2016

[8]. Hailu, A.; Rahman, S.: Evaluation of Key Success Factors Influencing ERP Implementation Success, Proc. of the 2012 IEEE 8th World Congress on Services, pp.89--91 (2012)

[9]. Hallikainen, P.; Kivijarvi, H.; Nurmimaki, K.: Evaluating strategic IT investments: an assessment of investment alternatives for a web content management system, Proc. of the $35^{\text {th }}$ HICSS, pp. 2977--2986 (2002)

[10]. Jiang, Z.; Jain, D.C.: A Generalized Norton-Bass Model for Multigeneration Diffusion. Management Sci. 58(10), 1887--1897 (2012)

[11]. Joomla! web site. www.joomla.org, Open Source Matters, Inc. Accessed 07/2016

[12]. Kapur, P.K.; Sachdeva, N.; Singh, O.: Generalized discrete time modelfor multi generational technological products. In: Proc. Intern. Conf. on Futuristic Trends on Computational Analysis and Knowledge Management (ABLAZE), pp.717--723 (2015)

[13]. Keller, A.; Hüsig, S.: Ex-ante identification of disruptive innovations in the software industry applied to web applications: The case of Microsoft's vs. Google's office applications, Technol. Forecast. Soc. Change 76, 1044--1054 (2009)

[14]. Lee, J.; Siau, K.; Hong, S.: Enterprise Integration with ERP and EAI, Communications of the ACM 46(2), 54--60 (2003)

[15]. Leina, Z.; Tiejun, P.; Guoyan, R.; Chengbin, F.: Development and Implementation of ERP/CRM System Based on Open Source Software to Small and Medium-sized Enterprise in China. In: ICICTA, 2008 , Vol. 2, pp. 725--730 (2008)

[16]. Liu, X.; Zhang, W.J.; Prasad, R.; Tu, Y.L.: Manufacturing Perspective of Enterprise Application Integration: the State of the Art Review. Int. J. Prod. Res. 46(16), 4567--4596 (2008)

[17]. Patel, S.K.; Rathod, V.R.; Parikh, S.: A Statistical Comparison of Open Source CMS. In: Trendz in Inform. Sci. and Comput. (TISC), $3^{\text {rd }}$ Intern. Conf., pp. 182--187 (2011)

[18]. Skulimowski, A.M.J. (Ed.): Trends and Development Scenarios of Selected Information Society Technologies [in Polish]. Progress \& Business Publishers, Kraków (2015)

[19]. Skulimowski, A.M.J.; Badecka, I.: Competition Boosts Innovativeness: A Quantitative Software Evolution Model. In: T. Theeramunkong et al. (Eds.) Proceedings of the Tenth International Conference on Knowledge, Information and Creativity Support Systems (KICSS 2015), Phuket, Thailand, November 12-14, 2015, pp. 433--446 (2015)

[20]. Skulimowski, A.M.J.; Badecka, I.; Golonka, D.: New trends in the technological development of CMS-based enterprise software for SMEs. In: Skulimowski, A.M.J. (Ed.), Trends and Development Scenarios of Selected Information Society Technologies, Progress \& Business Publishers, Kraków (2015)

[21]. Snedecor, G.W.; Cochran, W.G.: Statistical Methods. J. Educ. Behav. Stat. 19(3), 304-307 (1994)

[22]. Wieringa, R.J.; Daneva, M.: Six strategies for generalizing software engineering theories. Sci. Comput. Program. 101, 136--152 (2015)

[23]. WordPress web site, www.wordpress.org. Accessed 07/2016

[24]. Tripathy, P.; Naik, K.: Software evolution and maintenance: a practitioner's approach. Wiley \& Sons, Inc., Hoboken, New Jersey, p.393 (2015)

[25]. Zykov, V.S.: Integrating Enterprise Software Applications with Web Portal Technology. Proceedings of the 5th International Workshop on Computer Science and Information Technologies CSIT'2003, Ufa, Russia, Vol.1, Ufa State Aviation Technical University Editorial-Publishing Office, pp. 60--65 (2003), available from arXiv:cs/0607127 